\title{
An Inverse Scattering Approach Based on Inhomogeneous Medium Green's Functions for Microwave Imaging of Brain Strokes
}

\author{
Eda Konakyeri Arıc1 ${ }^{*}$, Ali Yapar ${ }^{1}$ \\ ${ }^{1}$ Department of Electronics and Communication Engineering, Istanbul Technical University, Istanbul, Turkey \\ *corresponding author, E-mail: edakonakyeri@yahoo.com
}

\begin{abstract}
In this study, an inverse scattering approach is investigated for the detection and imaging of an abnormal structure (a bleeding or a stroke) inside the human brain. The method is mainly based on the solution of an integral equation whose kernel is the Green's function of the inhomogeneous medium (corresponding to a human head model) which is obtained by a numerical approach based on Method of Moments (MoM). In this context, an inverse scattering problem related to the difference of healthy and unhealthy brain models is formulated and a difference function is obtained which indicates the region where the anomaly is located by solving this inverse problem. In order to reduce the reflection effects caused by the electromagnetic differences between the free space and the brain, a matching medium is used as the background space.
\end{abstract}

\section{Introduction}

Forward and inverse electromagnetic scattering problems related to inhomogeneous objects are important research subjects currently and the results of those researches are widely used practically in many fields such as remote sensing, geophysical exploration, biomedical and microwave imaging, etc. [1-5]. One of the most significant applications of electromagnetic scattering problems on biomedical area is detecting abnormal structures/tissues on organs in human body. Especially, detection of various abnormal structures like cancer and bleeding on brain and breast cancer imaging have become very hot research topics for last two decades and thus a huge number of studies have been contributed to the open literature [6-10].

Main principle on these problems is illuminating the structures that are desired to be imaged by some incident wave sources and detecting/imaging the abnormal structures by using the measured scattering fields from these structures. In addition to being an inhomogeneous structure, the brain tissues also have electromagnetic properties substantially different from the background medium. Therefore, it is difficult to analyze the scattering field for such a high contrast medium. On the other hand, one of the most significant issues in these applications is the penetration problem arising from high conductivities. Usually, the incident waves could not penetrate into high contrast and lossy media such as brain or breast if they are excited from the free space. Most of the incident power will be reflected back from the surface of the structure that is clearly not desired. This problem can be alleviated by choosing proper electromagnetic parameters for background (host) media. If the contrast between the inhomogeneous medium under test and the surrounding host medium decreased to an acceptable degree then it would be possible for the incident wave to penetrate the inhomogeneous region and to interact with the tissues to be imaged. Such appropriate host medium is called as "matching medium". It is shown in the literature that using matching medium for the purpose of reducing the undesired effects such as huge reflections and of having larger penetration depths is useful $[6,11,12]$. To some extent the penetration problem could be reduced by an appropriate matching medium, however the other parameters such as the location, the size and the electromagnetic properties of the abnormal structures inside the brain are also other factors that may affect the solution significantly. In summary, the problem of detecting and/or imaging of inhomogeneities such as tumors or bleedings inside another inhomogeneous region such as brain or breast is a very difficult and challenging problem. As it is expected, the difficulties arising from the physical structure of the problem directly affect the mathematical formulation, and require some new approaches. Thus, the problem at hand is also very open to new contributions for more comprehensive analyses.

In this study, an inverse scattering approach is investigated for the detection and imaging of an abnormal structure inside the human brain. The method is mainly based on the solution of an integral equation whose kernel is the Green's function of the inhomogeneous medium that corresponds to a human head model. A MoM based numerical calculation of this type of Green's function is presented in [5] for an arbitrary source-observation point pair. The problem considered here is formulated and examined in a two-dimensional (2-D) configuration since it would be much more convenient to present the preliminary results, first. Extension to three-dimensional (3-D) problem that is certainly the more realistic case left as a future work. Hence, a slice of Zubal head phantom [13] will be used as a 2-D head model. For this purpose, 2-D head model is considered as an inhomogeneous medium and the scattering field from head model is represented with an integral expression by using the inhomogeneous Green's function. This representation is basically the convolution of 
inhomogeneous Green's function with the incident field. Then, based on the subtraction of two integral representations of healthy and bleeding head models, problem is reduced to a Fredholm integral equation of the first kind that contains the contrast difference function as an unknown. The contrast difference function refers to the difference between object functions of healthy and bleeding head models. This equation is solved using Tikhonov regularization to calculate the contrast difference function. Section 2 is devoted to the mathematical formulation of the problem in terms of integral equations. Various examples supporting the effectiveness and the applicability of the method are given as numerical results in Section 3, while in Section 4 the conclusions are outlined. Time factor $\exp (-i \omega t)$ is used and omitted throughout.

\section{Analysis of Scattering Fields from Inhomogeneous Structures and Inverse Problem}

Consider the inhomogeneous structure illustrated in Figure 1. In this structure, the two-dimensional and bounded region D is a finite size, non-magnetic and inhomogeneous dielectric medium with electromagnetic parameters $\mu_{0}$ and $\varepsilon(x)$. Total field $u$ at any point $x$ is written as

$$
u(x)=u^{i}(x)+u^{s}(x)
$$

where $u^{i}$ and $u^{s}$ denote the incident and the scattering fields, respectively. The wave equation for the total field is

$$
\nabla^{2} u(x)+\bar{k}^{2}(x) u(x)=0
$$

where

$$
\bar{k}^{2}(x)=\left\{\begin{array}{l}
k^{2}(x)=\omega^{2} \varepsilon(x) \mu_{0} ; x \in D \\
k_{b}^{2}=\omega^{2} \varepsilon_{b} \mu_{0} ; \text { otherwise }
\end{array}\right.
$$

is the square of the complex wave-number defined in whole space. In (3), the region outside $\mathrm{D}$ has the electromagnetic parameters $\varepsilon_{b}$ and $\mu_{0}$. To obtain the scattering field, first the total field in (1) is substituted in (2). Then using

$$
\nabla^{2} u^{i}(x)+k_{b}^{2} u^{i}(x)=0,
$$

the inhomogeneous wave equation for the scattered field could be written as

$$
\nabla^{2} u^{s}(x)+\bar{k}^{2}(x) u^{s}(x)=-k_{b}^{2} v(x) u^{i}(x) .
$$

When the electric type Green's function for this structure is shown as $G(x, y)$, the scattering field can be expressed as

$$
u^{s}(x)=k_{b}^{2} \int G(x, y) v(y) u^{i}(y) d y
$$

where

$$
v(x)=\left\{\begin{array}{l}
\frac{k^{2}(x)}{k_{b}^{2}}-1 ; x \in D \\
0 ; \text { otherwise }
\end{array}\right.
$$

is the object function, whose support is the region $D$. Calculation of the Green's function appearing in (6) for arbitrary $(x, y)$ pair is a complicated problem whose solution is given in [5]. In this present study, the head model is illuminated by a line source which is located to an arbitrary point $y \in R^{2} \backslash D$. Therefore, Green's function for inhomogeneous structures in (6) is calculated numerically by means of formulation given in $[5,14]$, taking the singularity of the problem into account.

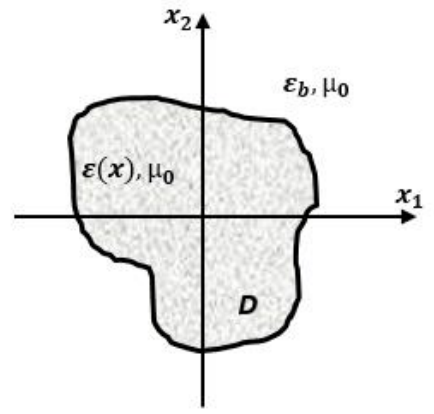

Figure 1: Geometry of the problem.

As the scattering field is obtained, the inverse problem can be examined. (6) is rewritten for healthy and bleeding brains as,

$$
\begin{aligned}
& u_{b b}^{s}(x)=k_{b}^{2} \int G_{b b}(x, y) v_{b b}(y) u^{i}(y) d y \\
& u_{h b}^{s}(x)=k_{b}^{2} \int G_{h b}(x, y) v_{h b}(y) u^{i}(y) d y .
\end{aligned}
$$

Now, subtracting (9) from (8) yields,

$$
\delta u^{s}(x)=k_{b}^{2} \int\left[G_{b b}(x, y) v_{b b}(y)-G_{h b}(x, y) v_{h b}(y)\right] u^{i}(y) d y
$$

Where $\delta u^{s}(x)=u_{b b}^{s}(x)-u_{h b}^{s}(x)$ shows the difference between scattering fields for healthy and bleeding brains. $G_{h b}$ and $G_{b b}$ denote the Green's functions for healthy and bleeding brains, respectively. It can be assumed that the bleeding area is not distinctly different from the general structure of the brain in terms of electromagnetic properties. Therefore, we can use the approximation $G_{b b}(x, y) \cong$ $G_{h b}(x, y)$. As a result, $(10)$ can be approximated as

$$
\delta u^{s}(x) \cong k_{b}^{2} \int G_{h b}(x, y) \delta v(y) u^{i}(y) d y
$$

where

$$
\delta v(y)=v_{b b}(y)-v_{h b}(y) .
$$

(11) is the difference between scattering fields from healthy and bleeding brains in terms of the contrast difference of bleeding and healthy cases given in (12). In order to detect the abnormal region inside the brain, calculating the difference between object functions of healthy and bleeding brains shown as $\delta v$ is sufficient. In other words, $\delta v$ is the contrast difference function. For this purpose, Fredholm integral equation of the second kind could be reduced to matrix system with the discretization that is expressed as

$$
[A][\delta v]=\left[\delta u^{s}\right] .
$$

In (13), $A$ shows the discretized matrix equivalent of integral operator which contains $G_{h b}(x, y)$ in $(11)$, while $[\delta v]$ and $\left[\delta u^{s}\right]$ correspond to the discretized versions of the related functions, respectively. Note that (13) (or equivalently (11)) is an ill-posed system since the kernel of the related integral operator is a compact one. Therefore, it would only be possible to obtain a regularized solution of (13). To this aim (13) is first reduced to the following regularized system 


$$
\left[\alpha I+A^{*} A\right][\delta v]=\left[A^{*}\right]\left[\delta u^{s}\right],
$$

where $A^{*}$ is the conjugate transpose of the matrix $A, I$ is the identity matrix in appropriate size and $\alpha$ is the Tikhonov regularization parameter. Then the solution in the sense of Tikhonov regularization is given by

$$
[\delta v]=\left[\alpha I+A^{*} A\right]^{-1}\left[A^{*}\right]\left[\delta u^{s}\right] .
$$

\section{Numerical Results}

In this study, with the purpose of detecting unhealthy regions inside the brain such as bleeding or tumor, a slice of Zubal head phantom is used as a 2-D head model [13]. Real and imaginary parts of relative dielectric permittivity of the head model are given in Figure 2(a) and 2(b). As it is seen in Figure 2(a) and 2(b), these values have high contrast in terms of electromagnetic parameters. The operating frequency is $1.2 \mathrm{GHz}$ and the head model is illuminated with 8 line sources which are surrounding it with distances of 0.2 and $0.28 \mathrm{~m}$ from the center. Observation points are $0.14 \mathrm{~m}$ by the center. The results are given by means of 2-D plots of real part of $\delta v$ which is the difference contrast. All results demonstrate arithmetic mean of the results obtained for each illumination.

It is observed from the numerical trials that in the single illumination case the results are not satisfactory. Therefore, in all numerical examples multiple illuminations ( 8 line sources separately) are used and quite satisfying results are obtained.
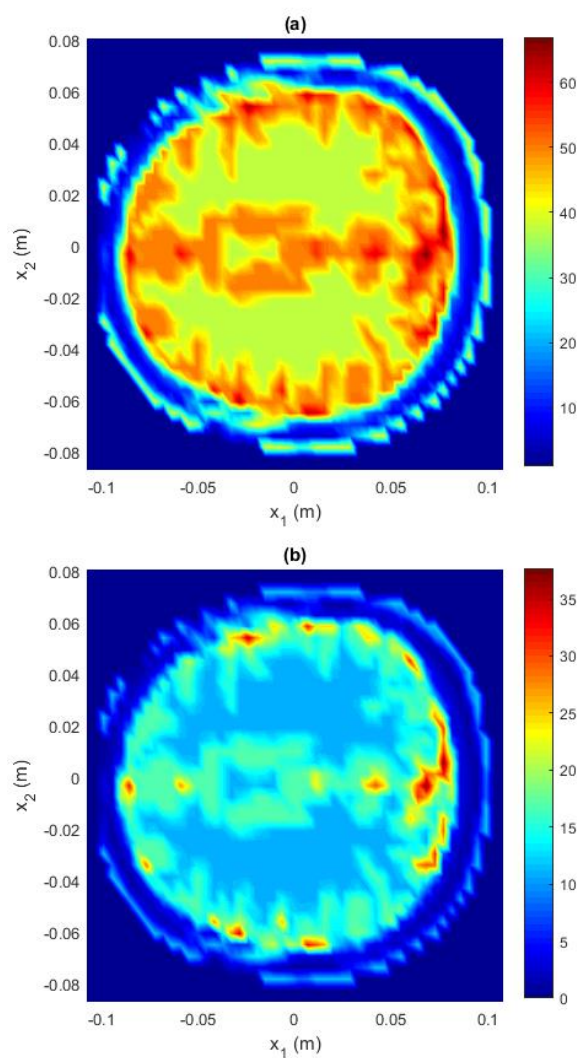

Figure 2: Variations of relative dielectric permittivity of 2-D head model (a) Real Part, (b) Imaginary Part.
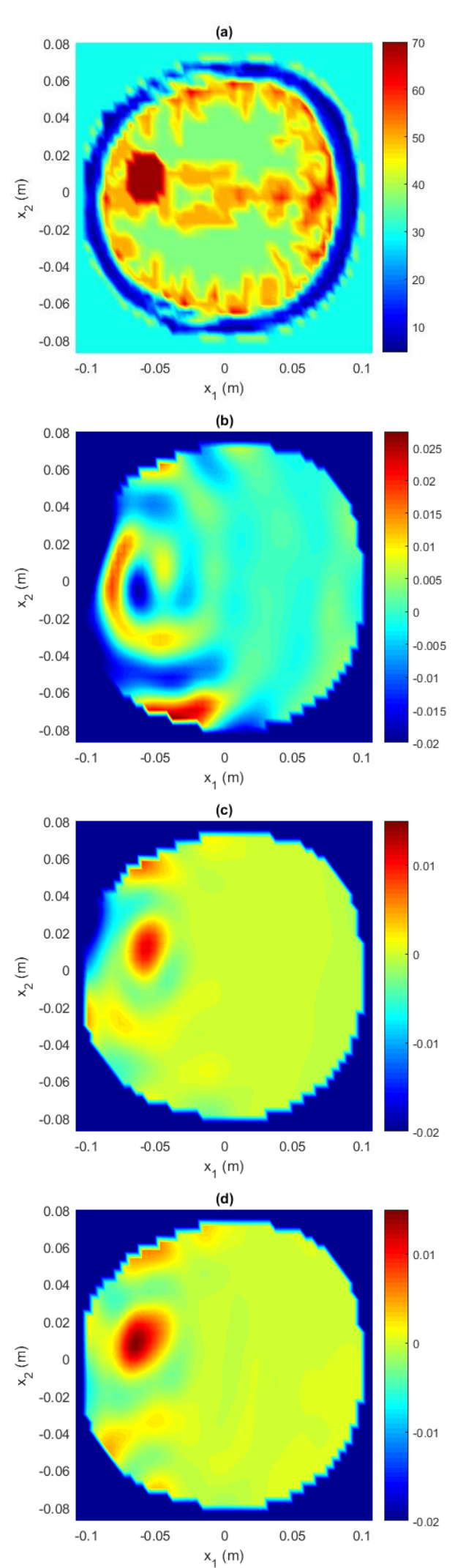

Figure 3: (a) Real parts of relative dielectric permittivity of 2-D head model with blood region, (b) Detection of blood region (Real parts of $\delta v$ ) without matching medium (MM), (c) with $\mathrm{MM} \varepsilon_{b r}=10$, (d) with $\mathrm{MM} \varepsilon_{b r}=30$. 

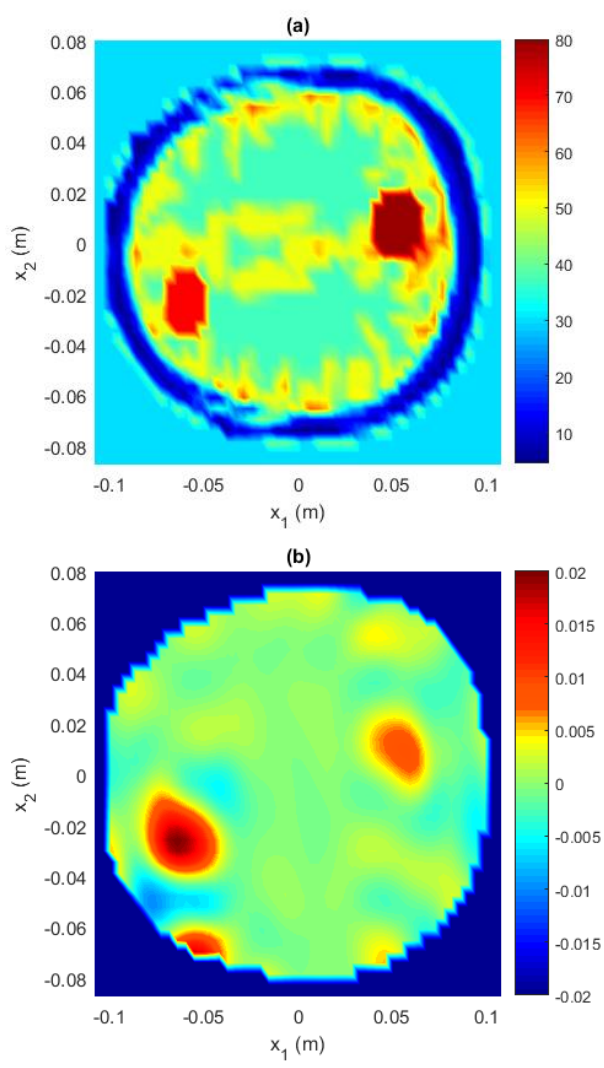

Figure 4: (a) Real parts of relative dielectric permittivity of 2-D head model with blood regions, (b) Detection of blood regions (Real parts of $\delta v$ ).

Firstly, an unhealthy head model given in Figure 3(a) is created by placing a structure with a relative dielectric permittivity of 70, (which is moderately different from electromagnetic parameters of the head model) into the head model. For the first application, the background medium is chosen as free space $\left(\varepsilon_{b r}=1\right)$. According to result given in Figure 3(b) that is obtained by using the proposed method, the blood region cannot be detected clearly. For an accurate analysis, as it is also explained in Section 1, incident field should penetrate into the head sufficiently. It is difficult for the incident field to penetrate into the brain due to the high contrast difference between the region outside the head and the head itself. As mentioned in Section 1, matching medium is used as a background medium to increase the penetrating field into the head during analysis $[6,11,12]$. To see the effect of matching medium for the proposed method, the analysis of the head model for $\varepsilon_{b r}=10$ is done in Figure $3(\mathrm{c})$. In this figure, the blood region is evident. As a result of the tests to determine the appropriate matching medium, it is determined that the optimal matching medium should have a relative dielectric constant $\varepsilon_{b r}=30$. The same analysis given in Figure 3(c) is repeated using matching medium with $\varepsilon_{b r}=30$ in Figure 3(d). Detection of the blood region inside the head model is accomplished easily by obtaining difference of object functions between two models in Figure $3(\mathrm{~d}) . \varepsilon_{b r}=30$ will be used in the following examples for background medium because the results are better than the other results used with different values of $\varepsilon_{b r}$ for this head model. The following examples are given to see if the method can detect blood region(s) when the location, size, number and dielectric permittivity of the bleeding are changed.
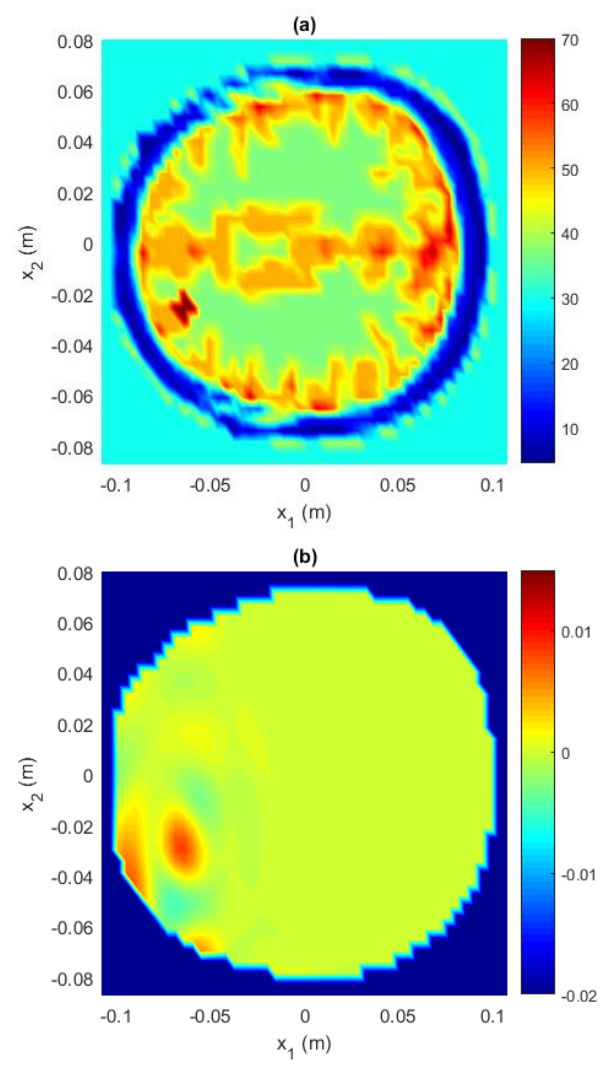

Figure 5: (a) Real parts of relative dielectric permittivity of 2-D head model with blood region, (b) Detection of blood region (Real parts of $\delta v$ ).

Two different structures are placed into the head model given in Figure 4(a) to see if the method can detect multiple and different blood regions in the brain. Relative dielectric constants of left and right blood regions are 70 and 80 , respectively. In Figure 4(b), detection of related regions is clearly seen.

In order to see the operability of the proposed method for bleeding which has smaller size than sizes of bleeding given in the other examples, a new small blood region in Figure 5 (a) with relative dielectric constant of 70 is considered. It is obvious from Figure 5(b) that the blood region is clearly detectable although it occupies a relatively small volume.

To examine the condition for relative dielectric permittivities of bleedings with smaller values that are close to the relative dielectric permittivity values of the head model, the bleedings are considered in Figure 6(a). There are three blood regions with relative dielectric constant of 50 . Because this value is quite close to the average values of the head model, blood regions are indicated by circles in order to make the bleedings clear. According to the results of Figure 6(b), the presented method can detect blood regions successfully for different types/sizes of bleeding. 

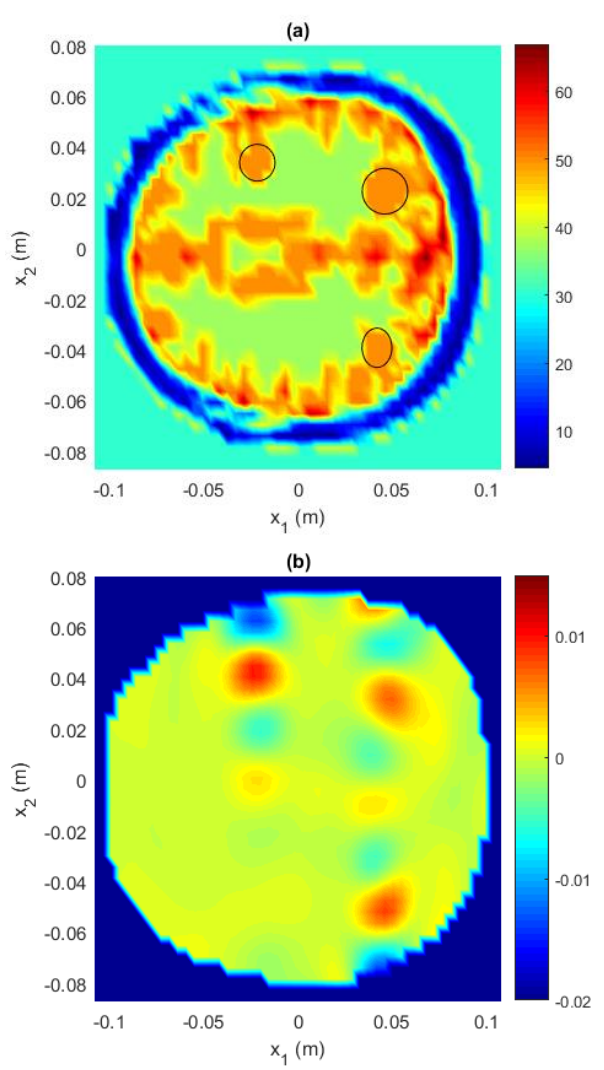

Figure 6: (a) Real parts of relative dielectric constant of 2-D head model with blood regions, (b) Detection of blood regions (Real parts of $\delta v$ ).

\section{Conclusions}

In this study, detection of abnormal regions such as a bleeding inside 2-D head model is successfully accomplished by means of proposed method. Matching medium should be chosen properly as a background medium in order to penetrate incident field into the head model and to obtain better results. The effectiveness of the method is analyzed through different examples. Electromagnetic properties, size, number and location of the blood region(s) inside the head model to be detected are important parameters for the applicability of the proposed method. Studies are being carried out to improve the method so the effect of these kind of differences on the problem could be reduced. Additionally, detection of the bleeding in 3-D head models, which would obviously be a more realistic case left as a future study.

\section{References}

[1] S.-W. Chen, H.-X. Zhou, K.-L. Zheng, W.-B. Kong, and W. Hong, VIE-ODDM-FFT method using nested uniform cartesian grid for the analysis of electrically large inhomogeneous dielectric objects, IEEE Transactions on Antennas and Propagation, Vol. 66, No. 1, pp. 293-303, January 2018.

[2] C. Yu, M. Yuan, Y. Zhang, J. Stang, R. T. George, G. A. Ybarra, W. T. Joines, and Q. H. Liu, Microwave imaging in layered media: 3-D image reconstruction from experimental data, IEEE Transactions on Antennas and Propagation, Vol. 58, No. 2, pp. 440-448, February 2010.

[3] N. Joachimowicz, C. Pichot, and J.-P. Hugonin, Inverse scattering: An iterative numerical method for electromagnetic imaging, IEEE Transactions on Antennas and Propagation, Vol. 39, No. 12, pp. 17421752, December 1991.

[4] G. Kobidze and B. Shanker, Integral Equation Based Analysis of Scattering From 3-D Inhomogeneous Anisotropic Bodies, IEEE Transactions on Antennas and Propagation, Vol. 52, No. 10, pp. 2650-2658, October 2004.

[5] E. Konakyeri Arıcı and A. Yapar, Numerical Calculation of 2-D Inhomogeneous Media Green's Function and Some Applications in Electromagnetic Scattering Problems, accepted to appear in IEEE Transactions on Antennas and Propagation.

[6] İ. Dilman, U. Yıldırım, S. Çoşğun, S. Doğu, M. Çayören and I. Akduman, Feasibility of Brain Stroke Imaging with Microwaves, 2016 IEEE Asia-Pacific Conference on Applied Electromagnetics (APACE), Langkawi, Malaysia, pp. 334-338, December 2016.

[7] U. Yıldırım, İ. Dilman, E. Bilgin, S. Doğu, M. Çayören and İ. Akduman, Continuous Monitoring of Hemorrhagic Brain Strokes via Contrast Source Inversion, 2017 11th European Conference on Antennas and Propagation (EUCAP), Paris, France, pp. 408-411, March 2017.

[8] T. U. Gürbüz, B. Aslanyürek, A. Yapar, H. Şahintürk, and I. Akduman, A nonlinear microwave breast cancer imaging approach through realistic body-breast modeling, IEEE Transactions on Antennas and Propagation, Vol. 62, No. 5, pp. 2596-2605, May 2014.

[9] E. C. Fear, X. Li, S. C. Hagness and M. A. Stuchly, Confocal microwave imaging for breast cancer detection: Localization of tumors in three dimensions, IEEE Transactions on Biomedical Engineering, Vol. 49, No. 8, pp. 812-822, August 2002.

[10] S. Y. Semenov, A. E. Bulyshev, A. Abubakar, V. G. Posukh, Y. E. Sizov, A. E. Souvorov, P. M. van den Berg, and T. C. Williams, "Microwave-tomographic imaging of the high dielectric-contrast objects using different image-reconstruction approaches", IEEE Transactions on Microwave Theory and Techniques, Vol. 53, No. 7, pp. 2284-2294, July 2005.

[11]P. M. Meaney, C. J. Fox, S. D. Geimer and Paulsen K. D., Electrical Characterization of Glycerin: Water Mixtures: Implications for Use as a Coupling Medium in Microwave Tomography, IEEE Transactions on Microwave Theory and Techniques, Vol. 65, No. 5, pp. 1471-1478, May 2017.

[12]R. Scapaticci, L. Di Donato, I. Catapano, and L. Crocco, A Feasibility study on microwave imaging for brain stroke monitoring, Progress In Electromagnetics Research B, Vol. 40, pp. 305324, 2012.

[13]I. G. Zubal, C. R. Harrell, E. O. Smith, Z. Rattner, G. Gindi and P. B. Hoffer, Computerized three-dimensional 
segmented human anatomy, Medical Physics, Vol. 21, No. 2, pp. 299-302, February 1994.

[14] J. H. Richmond, Scattering by a dielectric cylinder of arbitrary cross section shape, IEEE Transactions on Antennas and Propagation, Vol. 13, No. 3, pp. 334-341, 1965. 